\title{
Algoritmo para Decodificação e Fusão de Dados Correlacionados em Redes de Sensores sem Fio
}

\author{
Fernando Pujaico Rivera e Jaime Portugheis
}

\begin{abstract}
Resumo-O artigo propõe um algoritmo para decodificação de fontes correlacionadas seguido de uma regra de fusão dos dados estimados. Considera-se um cenário em que as fontes correlacionadas são codificadas separadamente sem conhecimento da correlação e são transmitidas em canais ruidosos ortogonais. Códigos LDGM curtos são utilizados para codificação de canal. A correlação das fontes é explorada num decodificador conjunto. O algoritmo proposto é uma extensão do algoritmo iterativo de decodificação bit-flipping e possui complexidade ligeiramente superior à decodificação independente. Isto o torna adequado num cenário de redes de sensores sem fio cujo centro de fusão possui recursos limitados. Resultados de simulação de desempenho comprovam a viabilidade do algoritmo e da regra de fusão e demonstram ganhos de codificação em relação à uma decodificação independente.
\end{abstract}

Palavras-Chave-Fontes correlacionadas, problema CEO, redes de sensores, algoritmo de decodificação bit-flipping.

Abstract-The paper proposes an algorithm for decoding of correlated sources followed by a fusion rule of the estimated data. A scenario in which the sources are encoded separately without knowledge of the correlation and are transmitted over orthogonal noisy channels is considered. Short LDGM codes are used for channel coding. The correlation of the sources is explored in a joint decoder. The proposed algorithm is an extension of the iterative bit- flipping decoding algorithm and has slightly higher complexity than independent decoding. This makes it suitable in a scenario of wireless sensor networks whose fusion center has limited resources. Performance simulation results prove the feasibility of both the algorithm and the fusion rule and demonstrate coding gains relative to independent decoding.

Keywords-Correlated sources, CEO problem, sensor networks, bit-flipping decoding algorithm.

\section{INTRODUÇÃO}

Há um interesse sempre crescente no estudo e projeto de redes de sensores sem fio dada uma grande gama de aplicações e desafios existentes [1]. Um possível cenário destas redes ocorre quando diversos sensores precisam transmitir de forma eficiente a mesma informação para um centro de fusão remoto [2], [3], [4], [5], [6].

Neste artigo iremos considerar o cenário descrito acima. Ele pode ser modelado pelo problema CEO (do inglês, Chief Executive Officer) [7]. Um CEO está interessado numa fonte que não pode ser observada diretamente. Diversos agentes (sensores) fazem observações ruidosas da mesma fonte que são codificadas separadamente sem conhecimento da correlação existente e são transmitidas em canais ruidosos para um centro

Fernando Pujaico Rivera está no Departamento de Comunicações, Faculdade de Engenharia Elétrica e de Computação, Unicamp, Campinas-SP. Email: fpujaico@decom.fee.unicamp.br

Jaime Portugheis está na Divisão de Tecnologia de Telecomunicações, Faculdade de Tecnologia, Unicamp, Limeira-SP. E-mail: jaime@ft.unicamp.br de fusão. Iremos considerar que os canais são ortogonais. Neste caso limitantes teóricos de transmissão de informação podem ser alcançados com separação entre a codificação de fonte e a de canal [8]. Uma abordagem para atingir estes limitantes seria usar códigos complexos de acordo com o teorema de Slepian-Wolf [9]. Entretanto, esta abordagem poderia ser proibitiva para um número muito grande de sensores. Outra abordagem seria usar somente codificação de canal e explorar a correlação existente numa decodificação conjunta.

Além de adotarmos somente codificação de canal, iremos considerar um cenário onde o centro de fusão possui recursos limitados [10]. Neste cenário é adequado considerarse algoritmos de decodificação com complexidade tratável. Sendo assim, propomos uma extensão do algoritmo bitflipping [11], [12] que utiliza a correlação entre as fontes para obter decodificação conjunta com melhor desempenho que da independente. Além disso, sua complexidade é ligeiramente superior à da independente. Após a decodificação de canal realiza-se uma regra de fusão baseada no critério MAP de decisão. Separação entre decodificação de canal e regra de fusão também foi considerada em [6].

$\mathrm{O}$ artigo é organizado como segue. A Seção II descreve sucintamente o modelo do sistema de comunicação. O algoritmo de decodificação conjunta proposto é explicado na Seção III. A Seção IV descreve um limiar teórico de desempenho para o caso de separação entre a codificação de fonte e a de canal. A regra de fusão MAP é apresentada na Seção V enquanto na Seção VI os resultados de simulação do desempenho dos sistemas são comparados. Finalmente, a Seção VII tece alguns comentários e conclusões.

\section{Modelo do Sistema}

A Figura 1 mostra o modelo do sistema de codificação distribuída e decodificação conjunta. Cada fonte gera uma sequência binária $\mathbf{u}^{i}=\left(u_{1}^{i}, \cdots, u_{k}^{i}, \cdots, u_{K}^{i}\right), i=1,2, \ldots, I$, de comprimento $K$. Iremos assumir que

$$
u_{k}^{i}=u_{k}^{0} \oplus e_{k}^{i}
$$

onde $\oplus$ denota uma soma módulo dois, as variáveis $u_{k}^{0}$ são identicamente distribuídas, $e_{k}^{i}$ são variáveis binárias mutuamente independentes com $P\left(e_{k}^{i}=1\right)=p_{i}$ e são independentes de $u_{k}^{0}$. A correlação entre $u_{k}^{0}$ e $u_{k}^{i}$ será denotada por $\operatorname{corr}\left(u_{k}^{0}, u_{k}^{i}\right)$, sendo igual a $\left(1-2 p_{i}\right)$. A correlação entre $u_{k}^{i}$ e $u_{k}^{j}$, para todo $i \neq j$, será denotada por $\operatorname{corr}\left(u_{k}^{i}, u_{k}^{j}\right)$, sendo igual a $\left(1-2 p_{i j}\right)$, onde $p_{i j}=p_{i}+p_{j}-2 p_{i} p_{j}$.

No que segue nós não utilizaremos os índices "i” por simplicidade. Cada fonte é codificada utilizando-se um código 


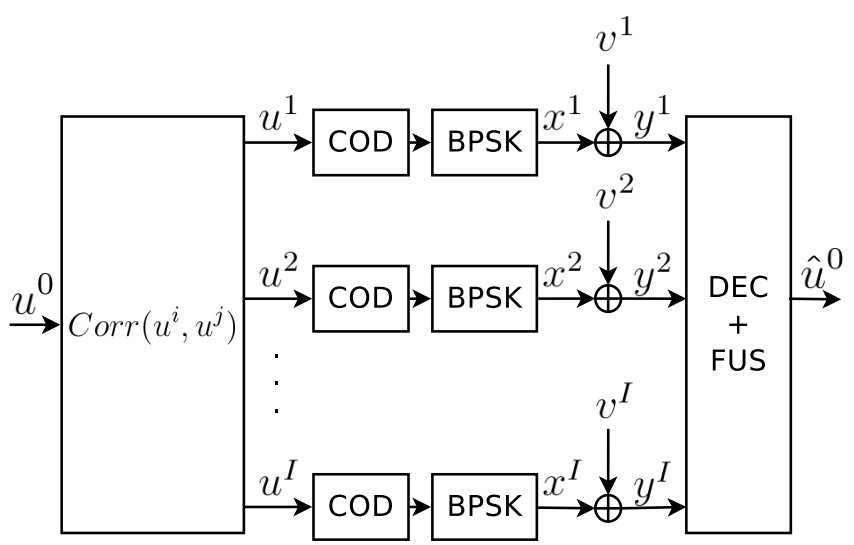

Fig. 1. Modelo do Sistema.

LDGM (do inglês "Low Density Generator Matrix") de taxa $R=K / N$, onde $N$ é o comprimento da palavra-código [13]. Cada palavra-código na saída dos codificadores é mapeada num vetor de sinalização $\mathbf{x}=\left(x_{1}, \cdots, x_{n}, \cdots, x_{N}\right), x_{n} \in$ $\{ \pm 1\}$, e é transmitido num canal com ruído aditivo Gaussiano branco. A entrada do decodificador no receptor é dada por $\mathbf{y}=\left(y_{1}, \cdots, y_{n}, \cdots, y_{N}\right)$. Cada componente de $\mathbf{y}$ é descrita pela soma $y_{n}=x_{n}+v_{n}, n=1, \cdots, N$ com $v_{n}$ sendo o ruído aditivo de média nula e variância $\sigma^{2}=\left(2 R E_{b} / N_{0}\right)^{-1}$. $\mathrm{Na}$ expressão da variância do ruído, $E_{b}$ é a energia por bit de informação e $N_{0}$ é a densidade espectral de potência do ruído considerada unilateral. Após aplicação de decisão abrupta no vetor $\mathbf{y}$, o vetor $\mathbf{z}=\left(z_{1}, \cdots, z_{n}, \cdots, z_{N}\right)$ é obtido.

\section{Algoritmo de DeCodifiCAÇÃo}

Nesta seção nós iremos considerar dois algoritmos distintos para obter uma estimação da informação transmitida por todas as fontes da Figura $1, \hat{\mathbf{u}}^{i}=\left(\hat{u}_{1}^{i}, \cdots, \hat{u}_{k}^{i}, \cdots, \hat{u}_{K}^{i}\right), i=$ $1,2, \ldots, I$.

Em ambos os algoritmos todos os decodificadores trabalham em paralelo. No primeiro algoritmo, nenhum dos decodificadores recebe informação dos outros decodificadores. Este algoritmo será referido como decodificação independente. Já no segundo algoritmo, todos os decodificadores compartem sua informação. Este algoritmo será chamado de decodificação conjunta.

\section{A. Decodificação Independente}

Para a decodificação independente iremos considerar o algoritmo proposto em [12] denominado "Parallel Hard BitFlipping" (PHBF). No que segue nós também não utilizaremos os índices "i" por simplicidade. Seja $H=\left[h_{m, n}\right]$ a matriz de verificação de paridade de um código LDGM. A $m$ ésima componente da síndrome é dada pela verificação $s_{m}=$ $\sum_{n} h_{m, n} z_{n}(\bmod 2)$. Denotaremos o conjunto de bits que participam na verificação $m$ e o conjunto de verificações nas quais o bit $n$ participa por $\mathcal{N}(m)=\left\{n \mid h_{m, n}=1\right\} \mathrm{e}$ $\mathcal{M}(n)=\left\{m \mid h_{m, n}=1\right\}$, respectivamente. Na decodificação PHBF a função de "flipping" é dada por

$$
E_{n}=\sum_{m \in \mathcal{M}(n)}\left(2 s_{m}-1\right) \text {. }
$$

O algoritmo completo PHBF é repetido abaixo [12].

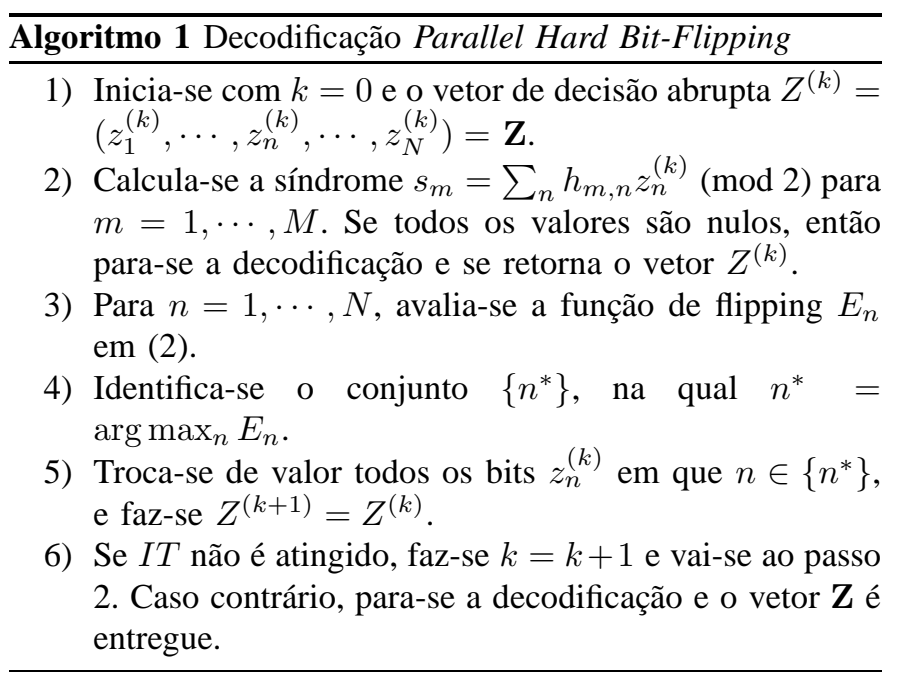

\section{B. Decodificação Conjunta}

O algoritmo de decodificação conjunta é obtido através da modificação da função de confiabilidade do algoritmo independente. A nova função de confiabilidade, $T_{n}^{i}$, será uma combinação da função $E_{n}^{i}$ dada em (2) com a função de confiabilidade conjunta, $C_{n}^{i}$. A função conjunta depende tanto dos bits recebidos de outros decodificadores quanto das correlações entre as fontes. A nova função para o $i$-ésimo decodificador será então dada por

$$
T_{n}^{i}=E_{n}^{i}+\left\lfloor\beta C_{n}^{i}\right\rfloor .
$$

onde $\lfloor b\rfloor$ é o maior inteiro menor ou igual a $b$ e $C_{n}^{i}$ é dada por

$$
C_{n}^{i}=\sum_{\begin{array}{c}
a=1 \\
a \neq i \\
z_{n}^{i}=z_{n}^{a}
\end{array}}^{I} \frac{E_{n}^{a} \operatorname{corr}(i, a)}{I-1}-\sum_{\begin{array}{c}
a=1 \\
a \neq i \\
z_{n}^{i} \neq z_{n}^{a}
\end{array}}^{I} \frac{E_{n}^{a} \operatorname{corr}(i, a)}{I-1}
$$

$\mathrm{O}$ parâmetro $\beta$ em (3) precisa ser otimizado. Se $\beta=0$, a decodificação conjunta se torna decodificação independente. Note que a introdução da operação de arredondamento $\lfloor b\rfloor$ leva a uma função de confiabilidade que opera somente com inteiros.

\section{LIMIAR TEÓRICO PARA CODIFICAÇÃo FONTE-CANAL CONJUNTA}

Como no cenário considerado neste artigo é válido o teorema da separação da codificação de fonte e da codificação de canal [8], pode-se combinar o Teorema de Slepian-Wolf [9] com o Teorema de Shannon para se obter um limiar teórico para a codificação fonte-canal conjunta como sugerido em [2],[3]. Sejam $R_{i}$ e $C_{i}, i=1,2, \ldots, I$, as taxas das fontes e as capacidades dos $I$ canais ortogonais, respectivamente. Sendo o teorema da separação válido, podemos escrever:

$$
R_{i} R_{c} \leq C_{i}, i=1,2, \ldots, I
$$


onde $R_{c}$ é a taxa de codificação de canal comum a todas as fontes.

Se somarmos os lados direito e esquerdo das equações 5 para $i=1,2, \ldots, I$ obtemos

$$
R_{c} \sum_{i=1}^{I} R_{i} \leq \sum_{i=1}^{I} C_{i}
$$

Como cada capacidade de canal é uma função monótona da razão sinal-ruído no canal, podemos obter um limitante inferior teórico para esta razão se substituirmos a soma das taxas $R_{i}$ por um limitante teórico. Um limitante inferior teórico para a soma das taxas pode ser obtido da última equação que define a região de Slepian-Wolf [9] e é dado pela entropia conjunta de todas as fontes. Sendo assim,

$$
R_{c} H\left(u^{1}, u^{2}, \ldots, u^{I}\right) \leq \sum_{i=1}^{I} C_{i}
$$

Note que a equação $\sum_{i=1}^{I} R_{i}=\theta$ é um hiperplano e o menor valor da constante $\theta$ deve satisfazer a região de Slepian-Wolf. O limiar teórico foi calculado para os cenários considerados na Seção VI e indicou valores de razão sinalruído negativas. Isto significa que mesmo sob uma potência de ruído maior que a de sinal, comunicação confiável ainda é possível. Mas para que isto ocorra, os códigos a serem utilizados podem ser longos. Como os códigos considerados neste artigo são curtos, eles mostram desempenho bem distante dos limiares teóricos. Por esta razão, estes limiares negativos não são mostrados nos resultados de simulação da Seção VI.

\section{A REGRA DE FusÃo}

A regra MAP para obter o estimador de $u^{0}, \hat{u}^{0}$, calcula a razão de probabilidades

$$
\phi=\frac{P\left(u^{0}=1 \mid \hat{u}^{1} \hat{u}^{2} \cdots \hat{u}^{I}\right)}{P\left(u^{0}=0 \mid \hat{u}^{1} \hat{u}^{2} \cdots \hat{u}^{I}\right)}
$$

e compara seu valor $\phi$ com a unidade. Se $\phi \geq 1$, decide-se pelo bit 1 . Caso contrário, decide-se pelo bit 0 . Suponha que $P\left(u^{0}=0\right)=P\left(u^{0}=1\right)=1 / 2$. Após alguma manipulação chegamos a

$$
\phi=t_{1} t_{2} \cdots t_{I}
$$

onde

$$
t_{i}= \begin{cases}\frac{1-p b_{i}}{p b_{i}} & \text { se } \hat{u}_{i}=1 \\ \frac{p b_{i}}{1-p b_{i}} & \text { se } \hat{u}_{i}=0\end{cases}
$$

O valor de $p b_{i}$ é obtido como segue

$$
p b_{i}=p_{i}+p d_{i}-2 p_{i} p d_{i}
$$

onde $p d_{i}$ é a probabilidade de erro na decodificação do bit da $i$-ésima fonte, ou seja, $p d_{i}=P\left(\hat{u}^{i} \neq u^{i}\right)$. Note que se $p_{i}$ é muito menor que $p d_{i}, p b_{i} \approx p d_{i}$. Mas de maneira geral $p b_{i}$ depende de $p_{i}$. Estaremos assumindo que os valores de $p_{i}$ podem ser estimados através dos valores das correlações $\operatorname{corr}\left(u_{k}^{i}, u_{k}^{j}\right)$ ou através das saídas dos decodificadores de cada canal como foi feito em [2].

\section{Resultados de Simulação}

\section{A. Algoritmo de Decodificação}

Com intuito de verificar a viabilidade e o comportamento do algoritmo de decodificação conjunta proposto, foram inicialmente obtidos resultados de simulação computacional para cenários com $I=3, I=5$ e $I=10$. Eles foram obtidos para o caso não codificado e para o caso em que um mesmo código LDGM curto com parâmetros $K=204$ e $N=306$ foi utilizado para codificar todas as fontes. Em todas as simulações o número máximo de iterações, IT, foi fixado em 15. No modelo de correlação das fontes para todos estes cenários fez-se $p_{i}=0,1$ para toda fonte $i$. O desempenho é sempre mostrado através da média aritmética das probabilidades de erro de bit de cada fonte (BER, do inglês "Bit Error Rate"). As Figuras mostram também um limitante inferior para a BER. Ele é o mesmo limitante proposto em [13] para a decodificação independente de códigos LDGM. Como no algoritmo de decodificação conjunta todos os decodificadores trabalham em paralelo, o limitante inferior permanece válido.

A Figura 2 compara o desempenho da decodificação independente com a da conjunta para um cenário com $I=3$ para o parâmetro $\beta=0,7,0,8,0,9$ e 1,0 . Também foram obtidos resultados para outras faixas de valores de $\beta$. Entretanto a faixa mostrada contem o valor de $\beta$ que leva ao melhor desempenho na faixa de razões sinal-ruído consideradas. Sendo assim, o valor ótimo para $\beta$ deve estar entre 0,7 e 1,0. É importante ressaltar que este valor ótimo depende da razão sinal-ruído. Na prática, um valor distinto de $\beta$ para cada valor distinto de razão sinal-ruído deveria ser utilizado. Pode-se notar que a partir de uma razão sinal-ruído de aproximadamente $3,7 \mathrm{~dB}$, a decodificação conjunta demonstra ganho quando comparada a um sistema sem codificação. Nota-se também que nesta região há ganho sobre a decodificação independente. Este ganho só irá diminuir para razões sinal-ruído altas. Esta observação continua válida para outros cenários como veremos a seguir.

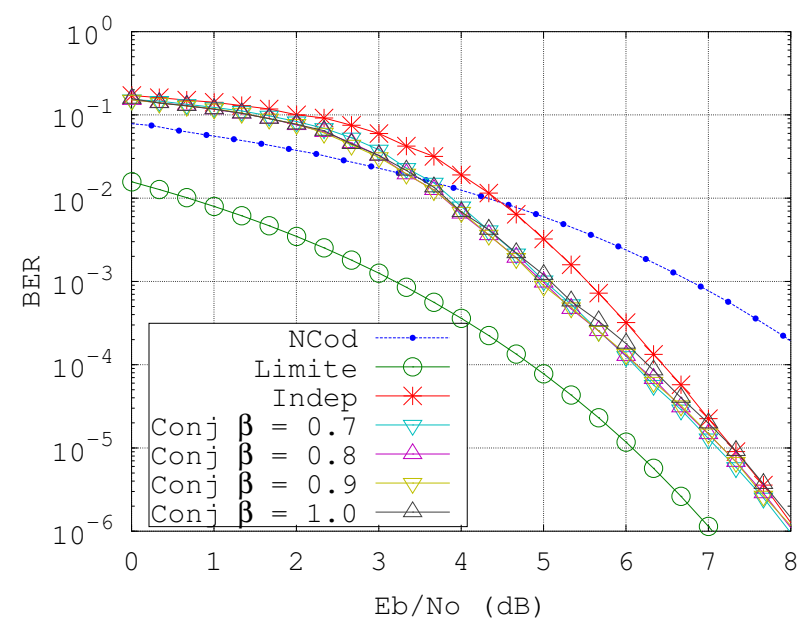

Fig. 2. BER para um sistema com 3 fontes utilizando decodificação independente ('Indep") e decodificação conjunta ("Conj"). O desempenho de um sistema sem codificação de canal (Ncod) e o limitante inferior (Limite) também são mostrados.

A Figura 3 compara o desempenho da decodificação independente com a da conjunta para um cenário com $I=5$. 
Agora o $\beta$ está entre 0,8 e 1,1 e já a partir de $3,3 \mathrm{~dB}$, a decodificação conjunta demonstra ganho sobre o sistema não codificado e sobre a decodificação independente.

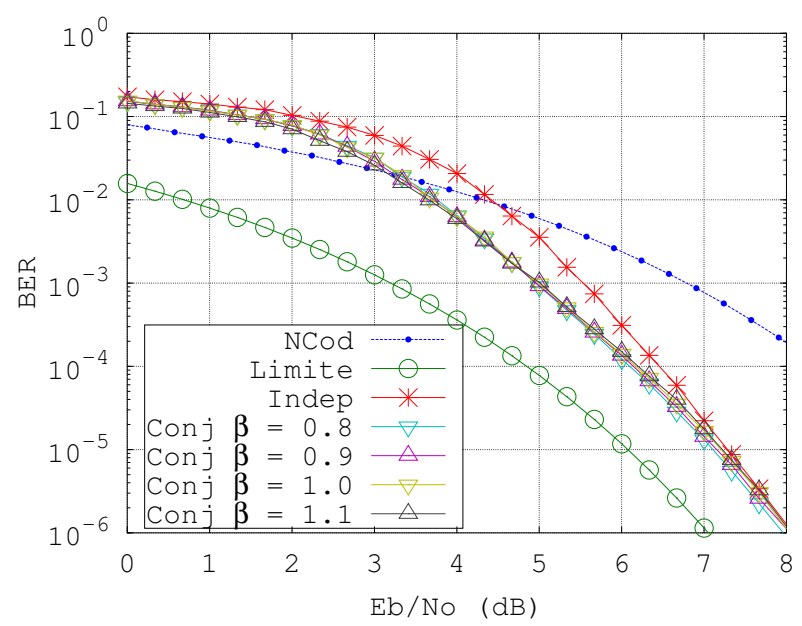

Fig. 3. BER para um sistema com 5 fontes utilizando decodificação independente ('Indep") e decodificação conjunta ("Conj"). O desempenho de um sistema sem codificação de canal (NCod) e o limitante inferior (Limite) também são mostrados.

Já a Figura 4 compara o desempenho para um cenário com $I=10$. A Figura mostra o desempenho para dois valores de $\beta$ : 1,0 e 1,5. A partir de $3,0 \mathrm{~dB}$, a decodificação conjunta demonstra ganho sobre o sistema não codificado e o independente. Aqui é importante ressaltar que quanto maior for o número de fontes consideradas no cenário menor será a razão sinal-ruído mínima para a qual o sistema com decodificação conjunta já apresenta ganhos de decodificação. A Figura 4 também mostra o desempenho para $I T$ fixado em 30. Não há variação de desempenho em relação à $I T=15$. Vale a pena notar também que neste cenário fica ressaltada a dependência do $\beta$ com a razão sinal-ruído: para $E_{b} / N_{0} \leq 4$, o $\beta$ de melhor desempenho vale 1,5 , enquanto que para $E_{b} / N_{0}>4$, o $\beta$ com melhor desempenho vale 1,0 .

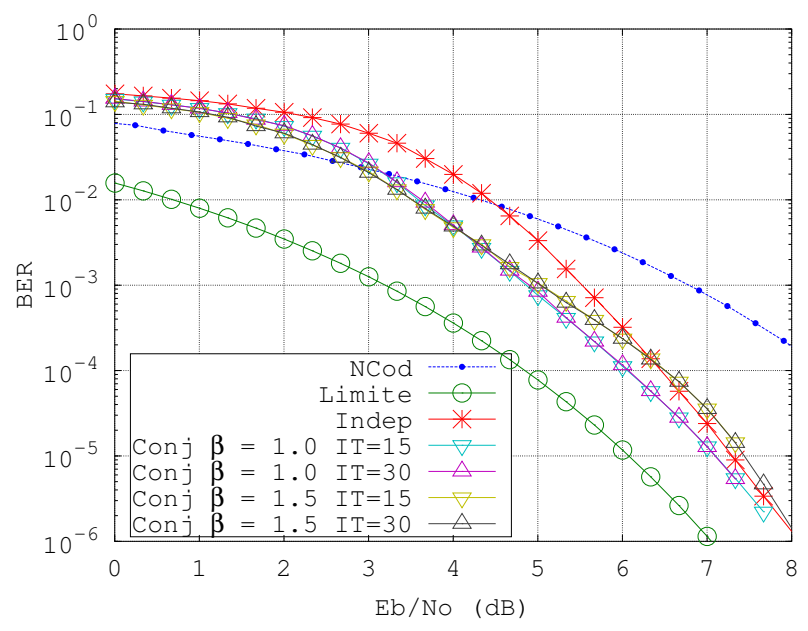

Fig. 4. BER para um sistema com 10 fontes utilizando decodificação independente ("Indep") e decodificação conjunta ("Conj"). O desempenho de um sistema sem codificação de canal (NCod) e o limitante inferior (Limite) também são mostrados.

\section{B. Regra de Fusão}

As Figuras 5 e 6 mostram resultados de desempenho para a regra de fusão. Eles foram obtidos para o caso não codificado e para o caso em que o mesmo código LDGM curto da subseção anterior foi utilizado para codificar todas as fontes. As Figuras mostram também um limitante inferior para a BER da fusão, considerando-se que não há ruído em todos os canais. Para a Figura 5, o limitante inferior foi obtido analiticamente em [2], [6]. Para o cenário da Figura 6, ele foi obtido através de simulação computacional.

A Figura 5 mostra resultados de desempenho para a regra de fusão considerando um cenário com 5 fontes e $p_{i}=$ $0,01, i=1, \ldots, 5$. É importante notar que o desempenho da decodificação independente é sempre inferior ao da conjunta como também ao do sistema não codificado. A partir de uma razão sinal-ruído maior que 3,7 dB há ganho da decodificação conjunta sobre o sistema não codificado. Mas este ganho não é significativo.

A razão de não mostrarmos resultados para cenários com $p_{i}=0,1$ para toda fonte $i$ (como na subseção anterior) reside no fato de que não se observa ganhos de desempenho em relação a um sistema não codificado. Esta é também a razão de encontrarmos na literatura resultados de desempenho para valores de $p_{i}$ menores ou igual a 0,01 [7], [5], [6].

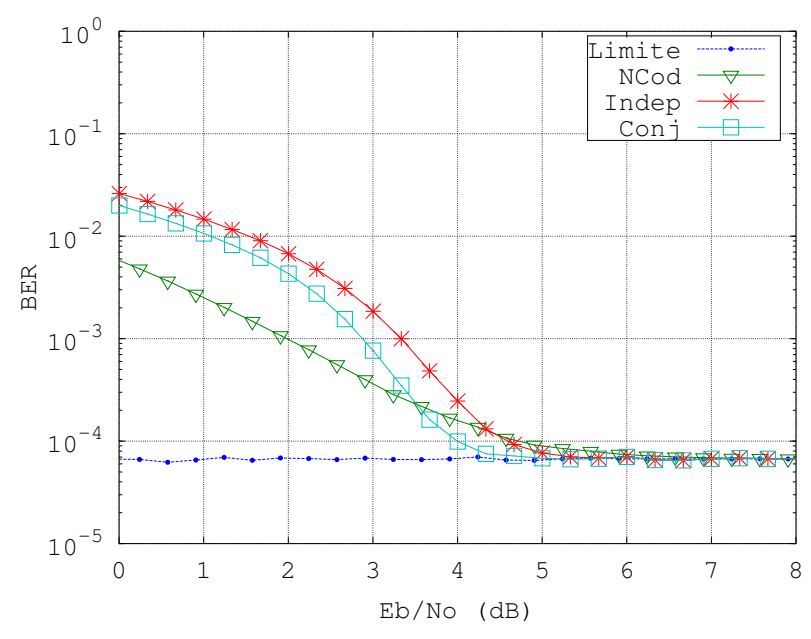

Fig. 5. BER da decodificação conjunta seguida da regra de fusão para um sistema com 5 fontes com $p_{i}=0,01, i=1, \ldots, 5$. O desempenho de um sistema sem codificação de canal (NCod) e o limitante inferior (Limite) também são mostrados.

A Figura 6 mostra resultados de desempenho para a regra de fusão considerando um cenário com 5 fontes e com $p_{1}=$ $0,001, p_{2}=0,12, p_{3}=0,19, p_{4}=0,4$ e $p_{5}=0,27$. O valor de $p_{1}$ foi selecionado intencionalmente e os outros quatro valores foram gerados aleatoriamente. Para uma BER igual a $3,0 \times 10^{-3}$, o ganho sobre o sistema não codificado é de 1,3 $\mathrm{dB}$ e sobre o sistema com decodificação independente é 0,4 dB.

\section{COMENTÁRIOS E CONCLUSÕES}

Resultados de desempenho de algoritmos de decodificação e fusão mostrados na literatura consideram quase sempre um 


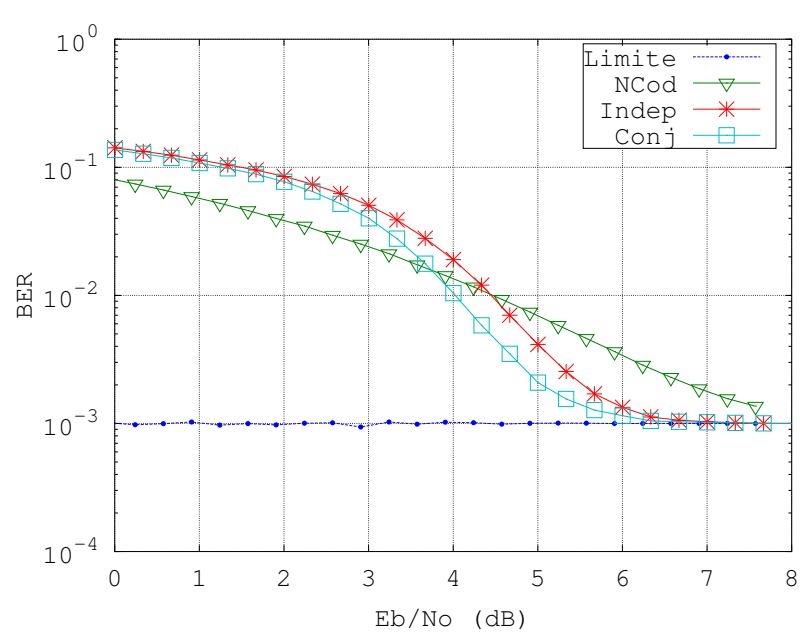

Fig. 6. BER da decodificação conjunta seguida da regra de fusão para um sistema com 5 fontes com distintos $p_{i}, i=1, \ldots, 5$. O desempenho de um sistema sem codificação de canal (NCod) e o limitante inferior (Limite) também são mostrados.

modelo de correlação em que $p_{i}=p$, para todo $i$, [2], [3], [4], [5], [6]. A justificativa desta consideração reside no fato de que assume-se que um grande número de sensores são implantados de maneira densa. Apenas em [10] foi considerado um modelo com valores de correlação gerados aleatoriamente. Entretanto, não foram obtidos resultados com uso de codificação de canal. Os resultados descritos na Figura 6 (para 5 sensores) indicam que se um dos sensores possui uma correlação muito alta com a fonte que não pode ser observada diretamente, ganhos de codificação significativos poderão ser obtidos. Um cenário com um número muito grande de sensores e com modelo de correlação aleatória está sendo atualmente investigado.

Os resultados de desempenho descritos neste artigo foram obtidos para um código de comprimento curto. Apesar do comprimento ser curto, a decodificação conjunta apresentou ganhos sobre a decodificação independente. Vale ressaltar que a complexidade da conjunta é ligeiramente superior à da independente. Uma investigação do desempenho não só de códigos mais longos como também de outras classes de códigos LDPC (do inglês, Low Density Parity Check Code) está em curso. Outro aspecto a ressaltar é que o algoritmo proposto é um algoritmo que trabalha com decisão abrupta. A extensão do algoritmo proposto para decisão suave também está sendo investigada.

\section{AgRADECIMENTOS}

Este trabalho foi parcialmente financiado pela FAPESP através do projeto 2012/22641-5. Os autores agradecem os comentários dos revisores que ajudaram a melhorar a apresentação final do artigo.

\section{REFERÊNCIAS}

[1] P. Corke, T. Wark, R. Jurdak, W. Hu, P. Valencia e D. Moore, Environmental Wireless Sensor Networks, Proceedings of the IEEE, vol. 98, no. 11, pp. 1903-1917, Nov. 2010.

[2] J. Del Ser, J. Garcia-Frias e P. M. Crespo, Iterative Concatenated Zigzag Decoding and Blind Data Fusion of Correlated Sensors, IEEE Int. Workshop on Scalable Ad Hoc and Sensor Networks, pp. 1-6, 2009
[3] K. Kobayashi, T. Yamazato e M. Katayama,Decoding of Separately Encoded Multiple Correlated Sources Transmitted over Noisy Channels, IEICE Trans. Fundamentals, vol. E92-A, no 10,pp 2402-2410, Out. 2009.

[4] J. Del Ser, J. Garcia-Frias, P. M. Crespo, D. Manjarres e I. Olabarrieta, On the Performance of Single LDGM Codes for Iterative Data Fusion over the Multiple Access Channel, MACOM 2010, LNCS 6235, pp. 47-57, Springer-Verlag 2010.

[5] A. Abrardo, G. Ferrari, e M. Martalò,On non-cooperative block-faded orthogonal multiple access schemes with correlated sources, IEEE Trans. Commun., vol. 59, no. 7, pp. 1916-1926, Julho 2011.

[6] G. Ferrari, M. Martalò, A. Abrardo, e R. Raheli,Orthogonal Multiple Access and Information Fusion: How Many Observations Are Needed?, in Proc. Inform. Theory and Applications Workshop (ITA), UCSD, San Diego, CA, USA, Fev. 2012.

[7] J. Haghighat, H. Behroozi, e D.V. Plant, Iterative joint decoding for sensor networks with binary CEO model, IEEE 9th Workshop on Signal Processing Advances in Wireless Communications, Julho 2008, Recife, Brazil.

[8] J. Barros e S. D. Servetto,Network information flow with correlated sources, IEEE Trans. Inform. Theory, vol. 52, no. 1, pp. 155-170, Jan. 2006.

[9] D. Slepian e J. K.Wolf, A coding theorem for multiple access channels with correlated sources, Bell Syst. Tech. J., vol. 52, no. 7, pp. 10371076, 1973.

[10] J. Barros e M. Tüchler, Scalable Decoding on Factor Trees: A Practical Solution for Wireless Sensor Networks, IEEE Trans. Commun., vol. 54, no. 2, pp. 284-294, Fev. 2006.

[11] Y. Kou, S. Lin e M. Fossorier, Low-density parity-check codes based on finite geometries: a rediscovery and new results, IEEE Trans. Inform. Theory, vol. 47, pp. 2711-2736, Nov. 2001.

[12] F. Pujaico e J. Portugheis, Algoritmos de Decodificação Abrupta para Códigos LDGM, XXX Simpósio Brasileiro de Telecomunicações, Brasília, pp. 1-6, Setembro, 2012.

[13] J. F. Garcia-Frias e W. Zhong, Approaching Shannon performance by iterative decoding of linear codes with low-density generator matrix, IEEE Commun. Lett., v. 7, n. 6, pp. 266-268, Junho 2003. 